\title{
EVALUATION AND ESTIMATION OF SOME GENETIC PARAMETERS FOR TOMATO HYBRIDS GROWN IN THE GREEN HOUSE
}

(Received:6.10.2014)

\author{
By \\ O. Kh. A. Al-Mfargy \\ Department of Horticulture and landscape, College of Agriculture, Diyala University, Iraq
}

\begin{abstract}
This study was conducted to evaluate the performance of five tomato genotypes, (Wajedan, Deena, Waad, Shahera and Nora). The experiment was carried out in the green house at two locations, the first in Baquba nursery and the second in Tomato Developing Research Center at Alghalibeeha for Directorate of Agriculture Diyala, Iraq during the growing season 2013-2014 in RCBD with three replicates. The studied characteristics were fruit number per plant, fruit weight average,yield per plant, TSS \% ,acidity percentage, fruit hardness degree and estimation of some genetic parameters for the genotypes.The results showed significant differences at $1 \%$ probability for most geneotype characteristics, except fruit weight average in both Locations, TSS\% at nursery Location under $5 \%$ probability. Genetic variation was more than environmental variation for all characteristics except TSS ratio in Alghalbeeha Location. Phenotypic variation coefficient values were close to genetic variation coefficient values at all characteristics except fruits TSS\% in Alghalbeeha Location. The results showed that the degree of broad sense heritability was high for all characteristics for both locations except fruit weight average in nursery location where it was medium, while TSS \% was low in Alghalbeeha location. Expected genetic improvement ratio as characteristics average was medium, except TSS\% and fruit weight average were low at both locations. Stable phenotypic values were high for fruits number per plant, fruit weight average, yield per plant and fruits hardness degree and there was low acidity ratio,TSS\% for all genotypes. Genetic parameters for the above characteristics were high for all genotypes except Wajedan and Waad, that as shown through reduction of their yields, while genetic parameter was reduced for TSS \%, acidity of fruits and hardness degree for Deena and Shaheera Genotypes.
\end{abstract}

Key words: genetic parameters, genetic resultant, tomato.

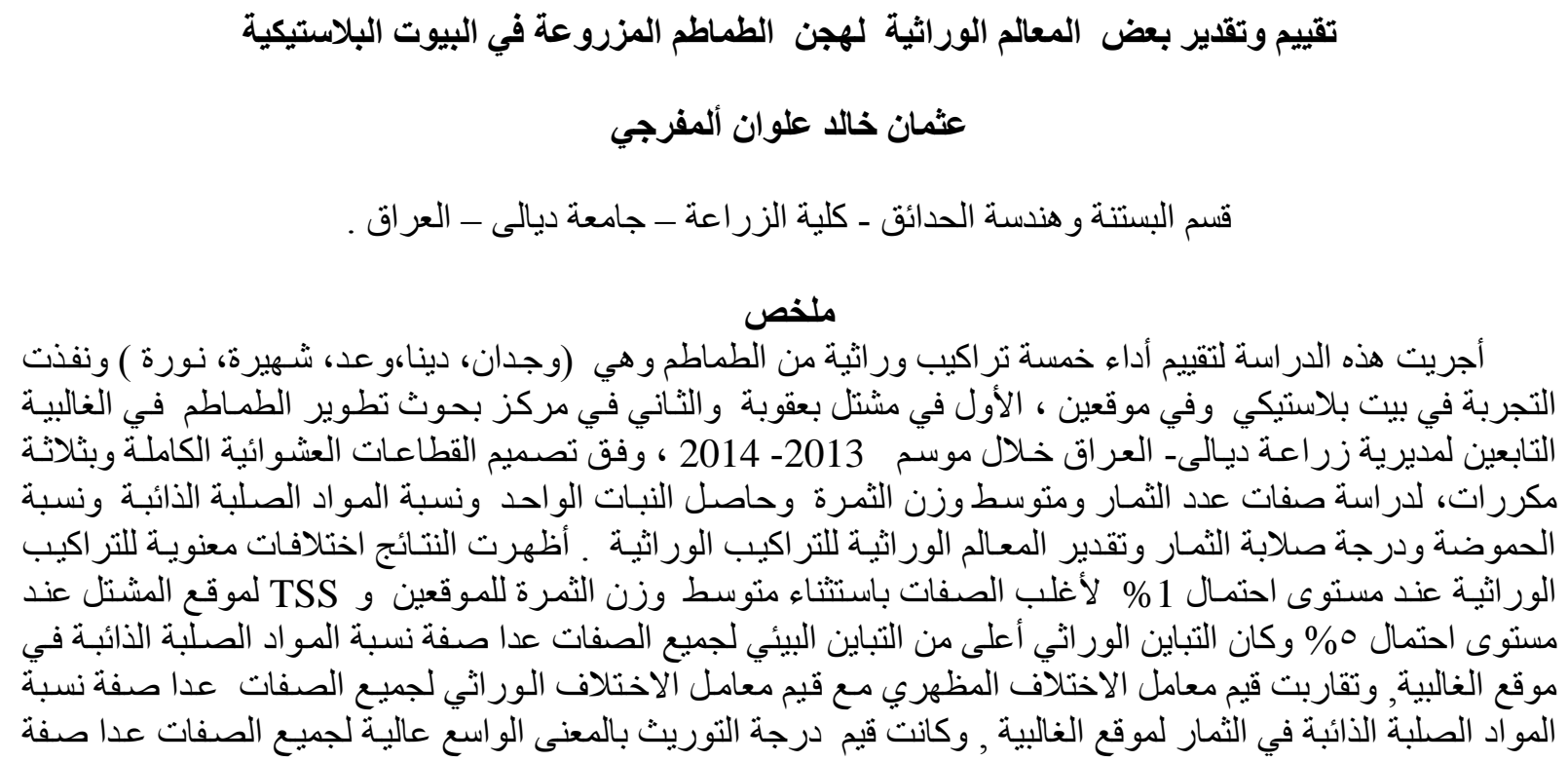


متوسطوزن الثمرة لموقع المشتل حيث كانت منوسطة في حين كانت منخفضة في صفة نسبة المو اد الصلبة الذائبة الكلية

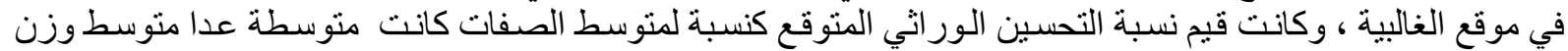

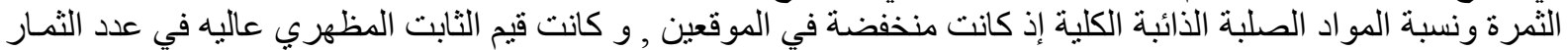

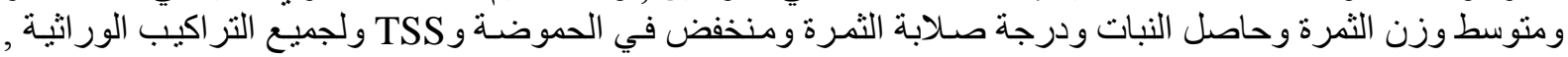

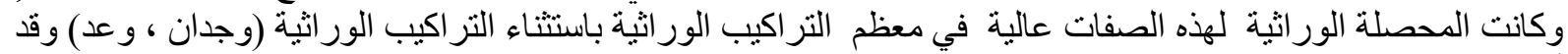

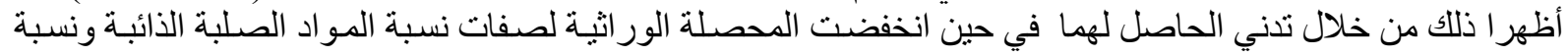
الحموضة ودرجة صلابة الثمار في التر اكيب الور اثية (دينا ، شهيرة الثين ).

ووجدت ألعبيدي ( 2012 ) في در استها حول تأثير

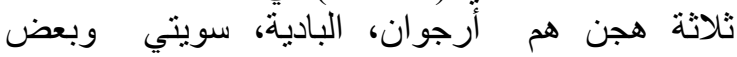

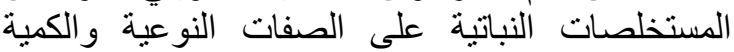
لحاصل الطماطة المزروعة في البيوت البهات البلاستيكية،

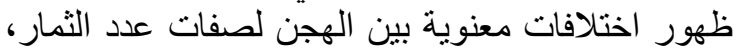

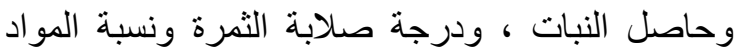

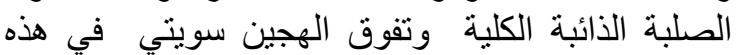

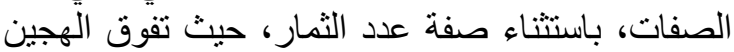

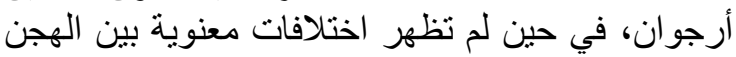
في صفات منوسط وزن الثمرة ونسبة الحموضة . ووجد) Osekita and Adedolapo 2014 عند در استهما لخمسة تر اكيب ور اثية من الطماطم المزرو عاتة في الحقل المكثوف للموسم الزربراعي 2011 2012 2012

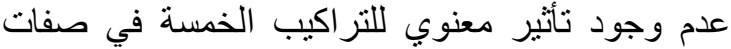
عدد الثمار ومنوسط وزن الثمرة ونير وكان معامل الاختلاف

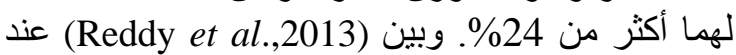

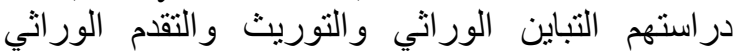

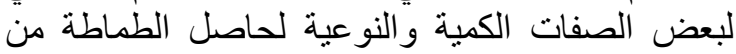

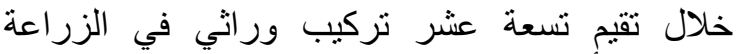

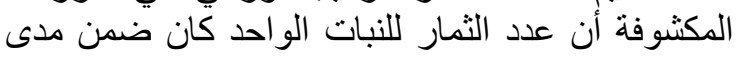

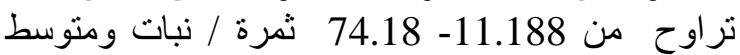
وزن الثمرة من 23.5- 118 102.33 غم / ثمرة وحاصل النبات الواحد كان من 0.013 -2.721 و النسبة المئوية للحموضة من من 0.179 \% - 0.879 \% ونسبة المو اد الصلبة الذائبة الكلية تراوحت بين الكبة

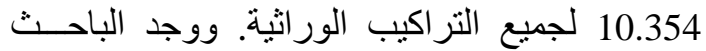
كند در استه لاختلافات الور اثية للجيل الأول من الطماطه خلال خريف

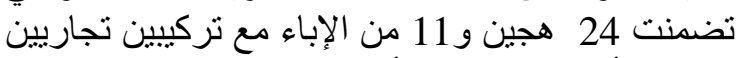
للمقارنة أن تحليل التباين أظهر اختلافات الات عالية المعنوية نجارين

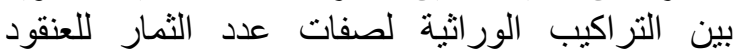

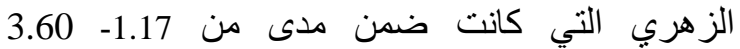

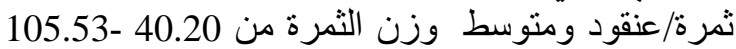
غم/ ثمرة وحاصل النبات من 1.00- 3.00 ـ 3.90 و الحموضة

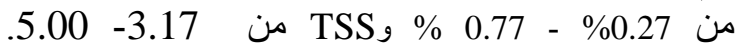
ووجد (Chernet et al.,2013) عند دراستهم للاختلافات الوراثية لصفات عدد الثمار وحاصل النبات

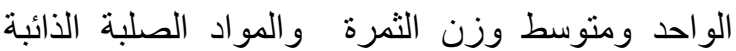
الكلية في ستة وثثلاثون تركيب ور الثي من الطي الطماطم

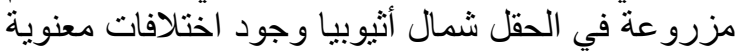

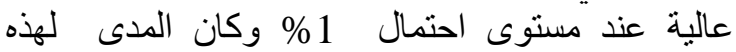
الصفات ( 4 - 9.7 ثمرة/ نبات، 0.3 - 2.10 كغم/ لهنا

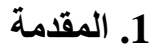

الطماطم (Solanum lycopersicon L.) نبات يتبع العائلة الباذنجانية Solanaceae ويعد من محاصيل

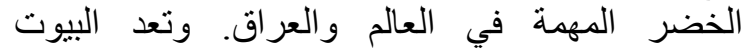
البلاستيكية المحمية من المنشآت الفعالة لزراعة الفئ وإنتاج

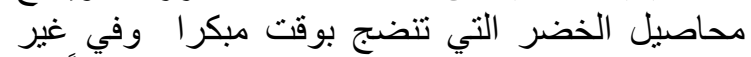

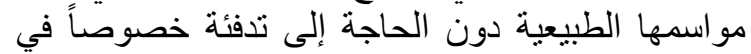

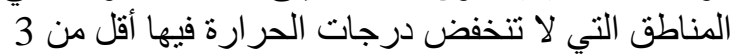
درجة مئوية. كما أنها تضاعف الإنتاجية في وحدة الإنة

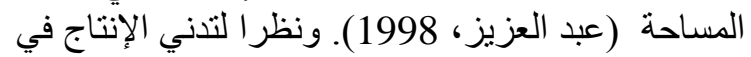

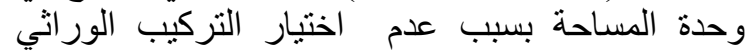

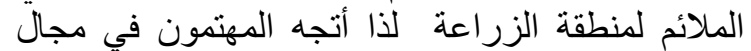
تربية وإنتاج الخضر إلى أجراء الخداء الأبحاث لتقيبيم أداء

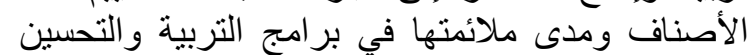

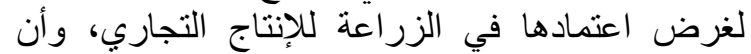

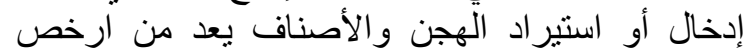
طرق التربية والتحسين الور اثي لاسيما في الدول الأنافية النامية

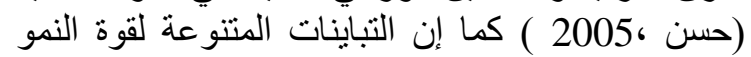

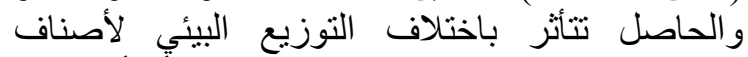

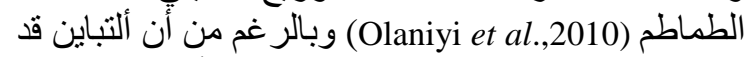

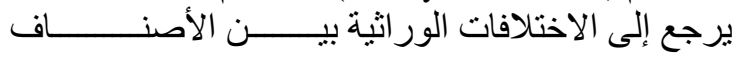

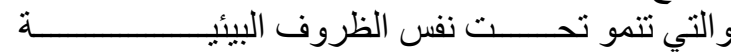
(Olaniyi and Fagbayade,1999) اختلافات وراثية واسعة ضمن كل من الهجن والأصناف

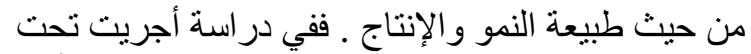

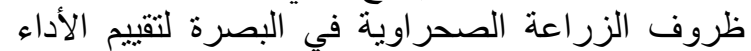

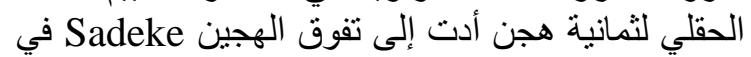
Sadeke ، Hatouf صفة وزن الثمرة وتفوف الهجين الهينان

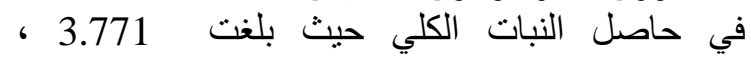

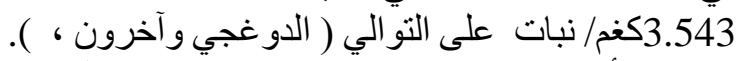

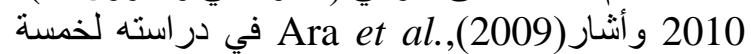

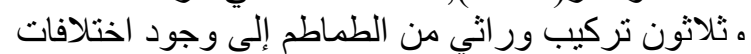

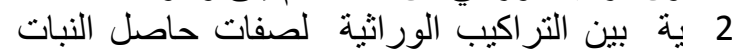

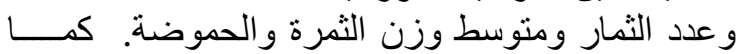

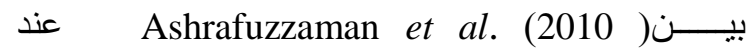
دراستهم تأثير ثمانية نر اكيب وراثية في تجربة حقلية لثلاثة مواسم مختلفة الظروف تركية البيئية على صئي صفات عدد

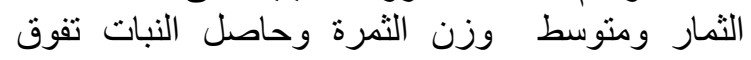

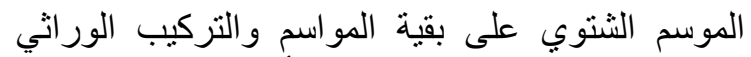

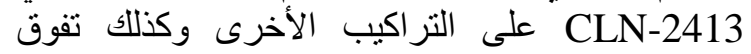
تداخلهما على بقية التداخلات . 
ووجد (Shankar et al.,2013) عند دراستهم للتغاير الوراثي للجيل الأول من الطماطم خلال خريف 2010 و التي تضمنت 24هجين و و11 من الإباء مع تركيبين

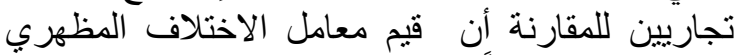
والور اثي كان عالياً وأن قيم

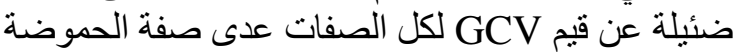

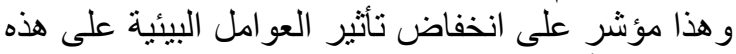

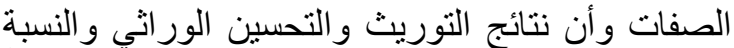
المئوية للتقدم الور اثتي من المنوسط العام للصفة كان التهان عالياً لكل الصفات و التي تؤشر إلى إمكانية استخدام الانتخاب التئي

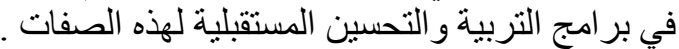

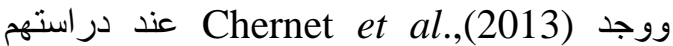
للتغاير ات الور اثثية لصفات عدد الثمار و الحاصل للنبات

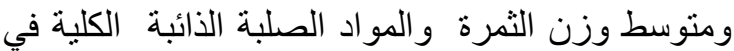

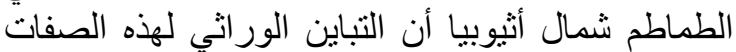

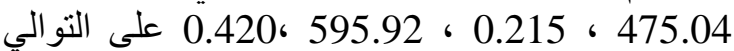
وكان يشكل الجزء الأكبر من التباين المظهري ولذائلكي

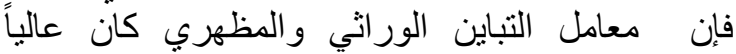
للصفات المدروسة باستثناء صفة المواد الصلبة الذائبة

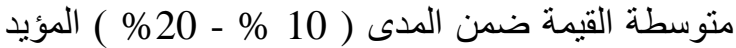

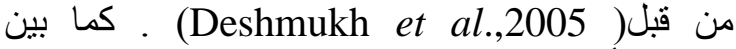
الباحثين أن درجة التوريث بالمعنى الواسع تراوحت من لون

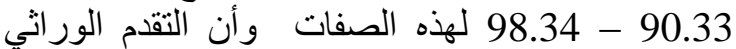
كنسبة مئوية من منوسط الصفة عند شدة انتخاب إذ كان 160.30\% ، 102.45 \% \% 102.

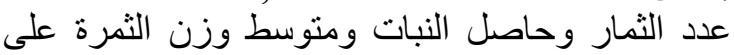

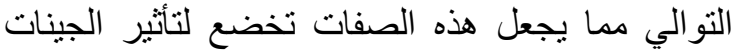

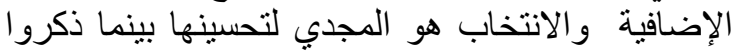

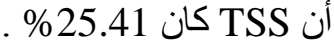
إن تقدير الإستقرارية والتأقلم للتركيب الوراثي في

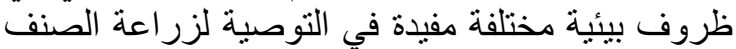

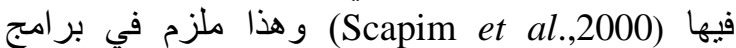
التربية. وفي دراسة أجريت على عشرين نركيب ور انئي

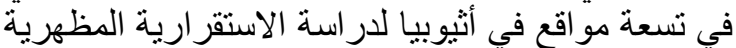

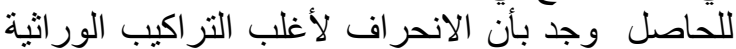

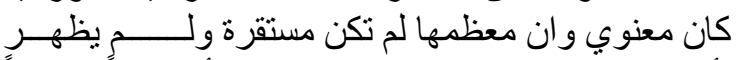

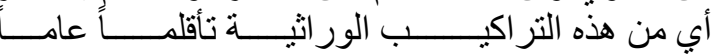
(Worku et al.,2001)

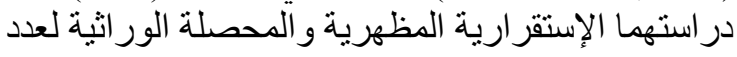

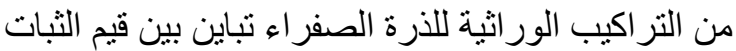

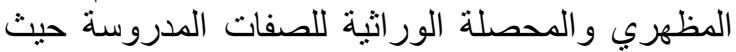

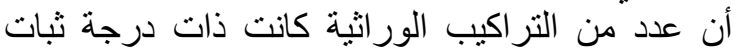

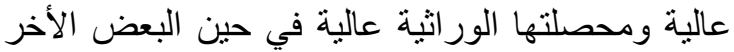
كان ذات قيمة ثبات عالية إلا أن محصلتها النية الوراثية الأنية

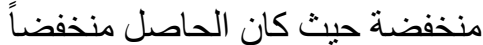

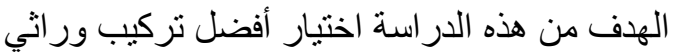

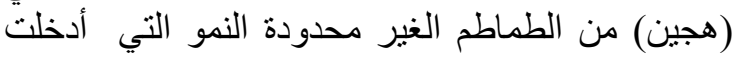

نبات، 18- 147 غم/ثمرة، 3.58-6.71 \% \%)، على أن قياس نسبة درجة التوريث بالمعنى الواسع و التي إئي

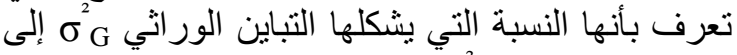

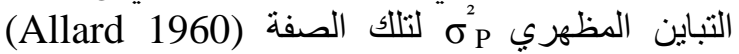
وتشمل درجة التوريث بهذا المعنى جميع أثنكال الفعل

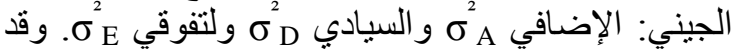

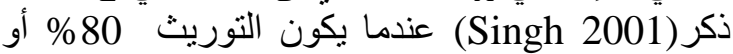
أكثر من السهل انتخاب هذه الصفات لأنه سيكون هناك الكئ تطابق وثيق بين التركيب الجيني والتركيب التئي الوراثي

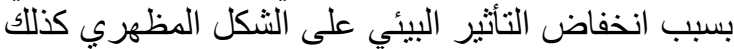

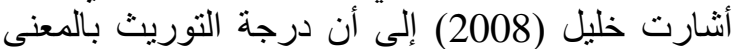

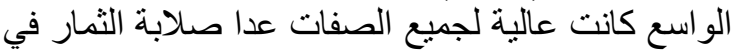
محصول الطماطة المزروعة في البيوت البلاستيكية .

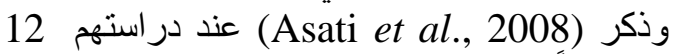
تركيباً ور اثياً من الطماطة ارتفاع قيم معامل التباين الوراثي و التوريث و والتقدم الور اثي لصفات أتفات عدد الثمار

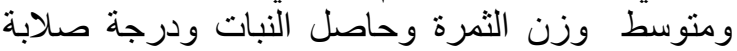

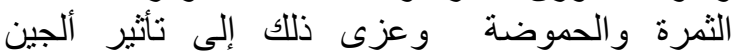

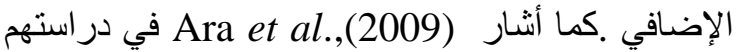

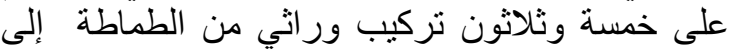
ارتفاع معامل التباين الور اثي و التوريث و والذي الذي تر افت مع الطي تعاظم قيم التحسين الور اثي لهذه التين الصفات. ووجد كل من(Osekita and Adedolapo 2014) عند دراسة لخمسة نراكيب وراثية من الطماطم

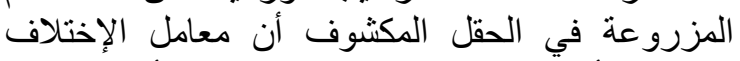

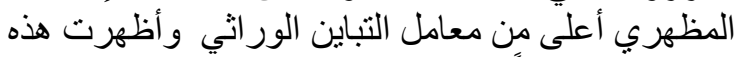

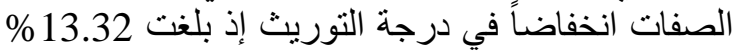

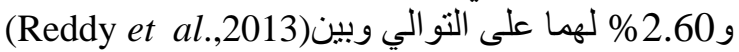
عند دراستهم التباين الور اثي والتي والتوريث و التقدم الور اثي

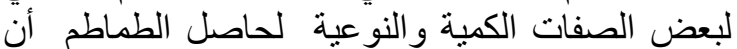

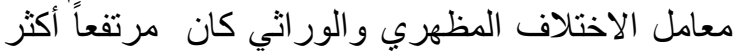

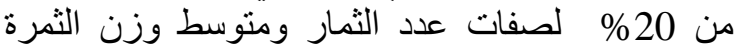

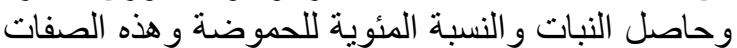
ممكن تحسينها بالانتخاب. بينما كان معامل الاختلاف

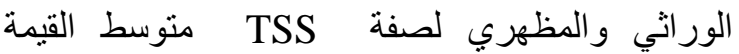
من10 -20\%. وبشكل عام كان معامل التباين الور اثي لتي

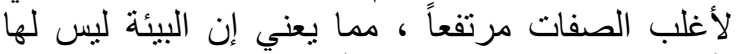

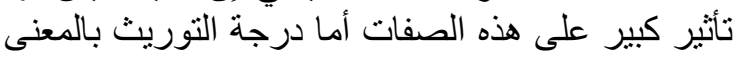

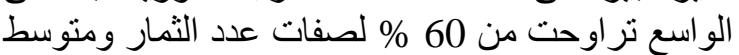

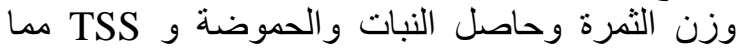
بعني أن الصفات ذات دات درجة التوريث التبات العالية أقل تأثر

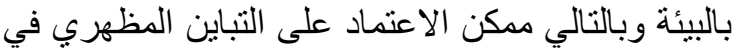

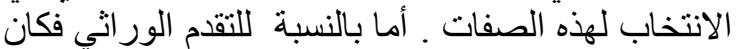

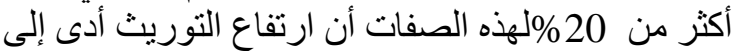

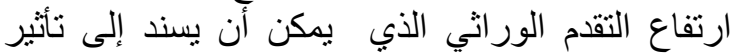
الجينات المضافة و عليه ممكن تحسين الصفات النين بالاتنخاب التير 
4.2. نسبة المواد الصلبة الذائبة الكلية (\% TSS) قيست بجهاز Hand-Refractometer بأخذ قطرة

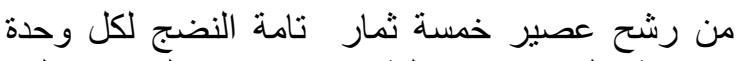

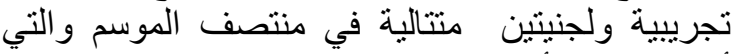
أجريت عليها أيضا قياسات صفات درجة الصنابة ونسبة

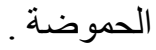

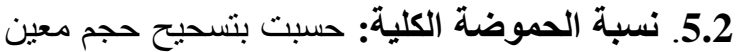

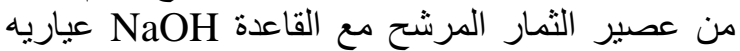

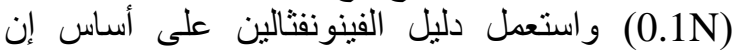

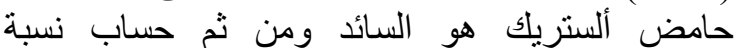

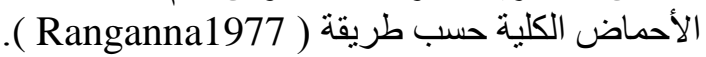
6.2. درجة صلابة الثمار( كغم /سم² :- قيست بواسطة جهاز Pressure tester 7.2.التحاليل الإحصائية والوراثية:

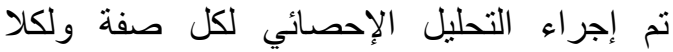
الموقعين باستخدام تصميم القطاعات العشوائية الكاملة

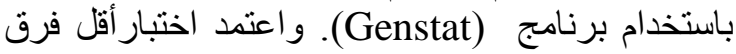

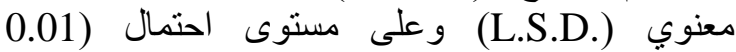
و0.05) للاستدلال على معنوية الفروق بين المتوسطات المان

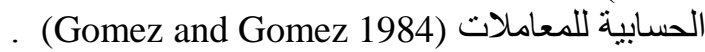
قدرت المعالم الور اثثية كما يلي: $\sigma_{G}^{2}=($ Mst - Mse)/r التباين الور اثني التئي $\sigma_{E}^{2}=$ Mse التباين البيئي $\sigma_{\mathrm{P}}^{2}=\sigma_{\mathrm{G}}^{2}+\sigma_{\mathrm{E}}^{2} \quad$ التباين المظهرين البنئ حيث إن: Mse = متوسط المربعات للخطأ التجريبي و

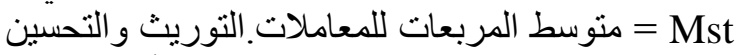

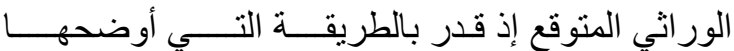
( Hanson et al., 1956) .

$$
H_{B . S}^{2}=\frac{\sigma_{G}{ }^{2}}{\sigma_{P}{ }^{2}}
$$

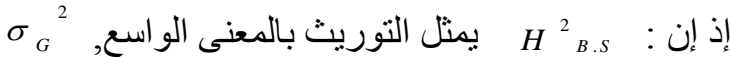
التباين الور اثي، ${ }^{2}$

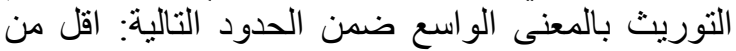

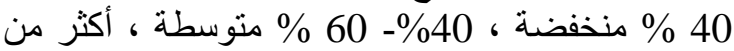

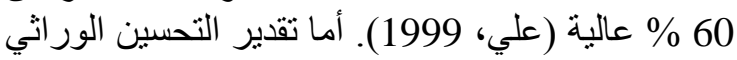

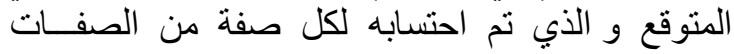
المدروسة باستخدام المعادلة التالية:-

$$
G . A=K . H^{2}{ }_{B . S} . \sigma_{P}
$$

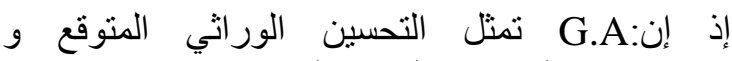

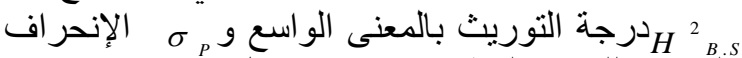

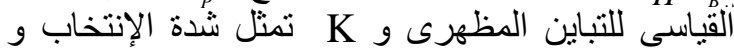
يساوى1.4 عند إنتخاب 20\% من النباتات وقدر التحسين

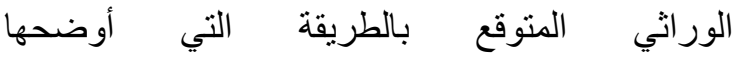
(Kempthorne,1969)
حديثا للعراق وذللك بتقدير معالمها الوراثية و ومدى إثى إمكانية إدخالها في برامج التربية لتربية واختيار أفضلها ل اللزر اعة المحمية .

\section{2.المواد وطرائق البحث}

نفذت التجربة في بيت بلاستيكي في موقعين من البه

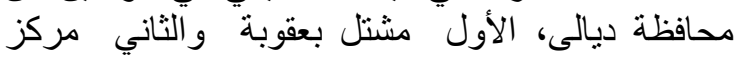
بحوث تطوير الطماطم في الغالبية التابعين لمديرية

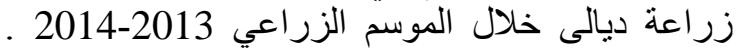

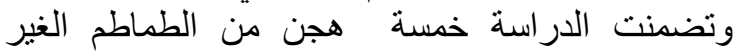
محدودة النمو هي ( وجدان من شركة بيتو سيد الأمريكية

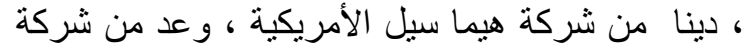

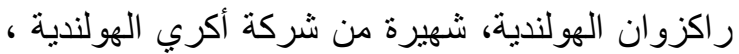

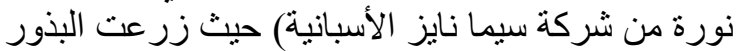

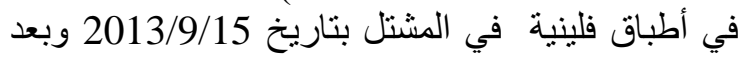

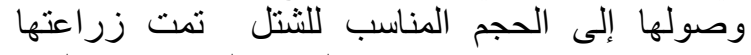
بتاريخ 1 /2013/11 في البيت البلاستيكي المعد فئن

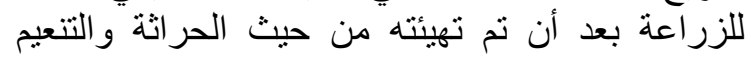
و التعديل وتعقيم التربة بالمبيدات القيت الفطرية (رايدوميل محبب) و إضافة الأسمدة العضوية المتحللة للتربة بكمية

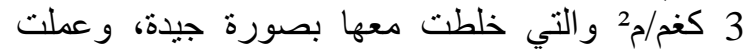

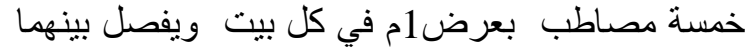
ممرات بعرض نصف متر وزرعت في الثتلات بخطين

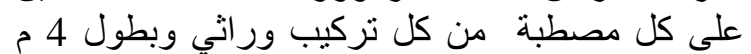
و المسافة بين النباتات 40 سم. وكانت مستاحة كل كل وحدة

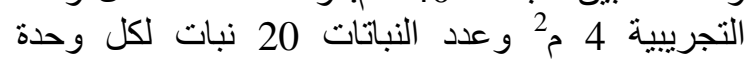

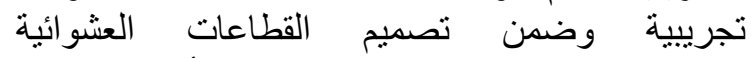

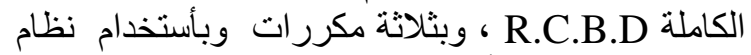

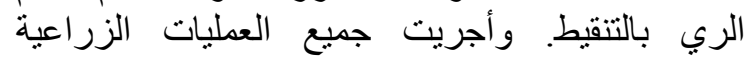

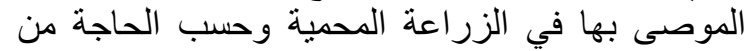
ري وتسميد وتسليق النباتات وإزالة الأفرع النالة الجانبية

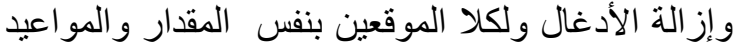

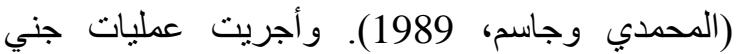
الحاصل بعد وصول الثمار إلى مرحلة النضج البـات البتاني

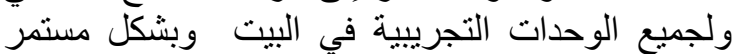

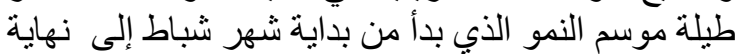

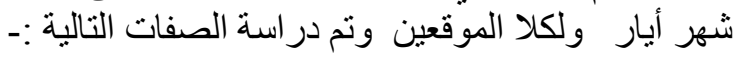

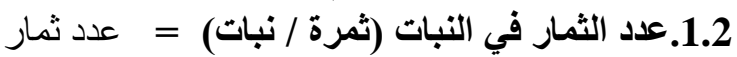
الوحدة التجريبية /عدد النباتات فيهات

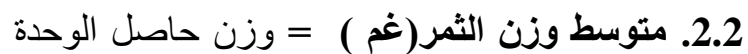
التجريبية / عدد الثمار فيها .

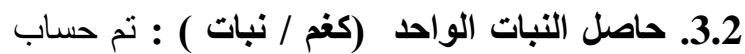

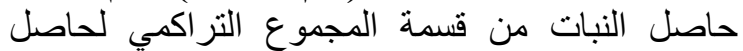
جميع الجنيات في كل وحدة تجريبية على عدد النباتات فيها. 
الوراثية عند مسنوى 1\% لجميع الصفات ولكلا الموقعين باستثناء صفات متوسط وزئ وزن الثمرة للموقعين

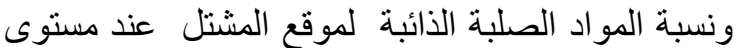

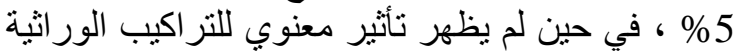

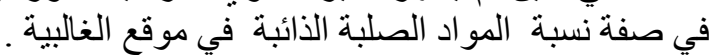

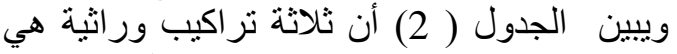

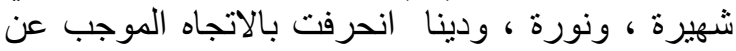

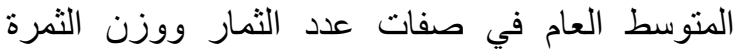

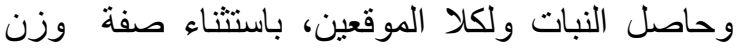
الثمرة للتركيب الوراثي دينا لموقع المشتل. وقد تفوق

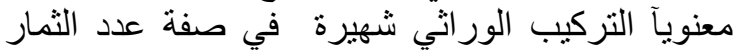
على بقية التراكيب إذ سجل 83.67 ، 83.00 ، 83.0

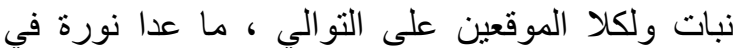

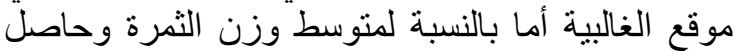

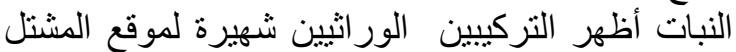

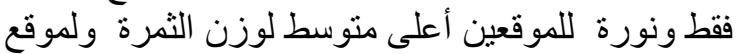

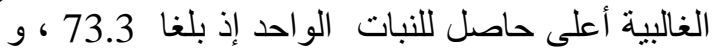

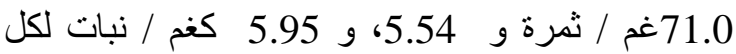

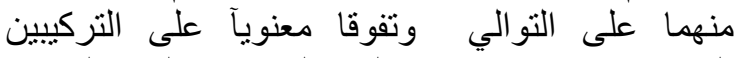
الور اثيين وجدان وو عد ولكلا الموقعين ولم يختلفا عن عنيا

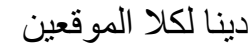

أما بالنسبة للصفات النوعية فقد انحرف تركين التيان

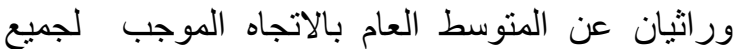

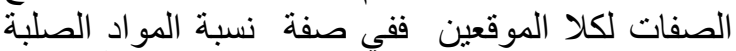

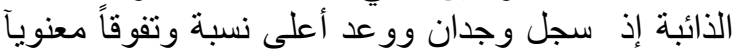

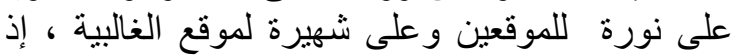

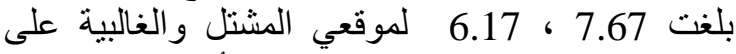

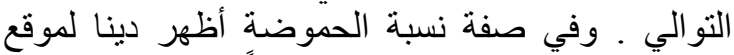

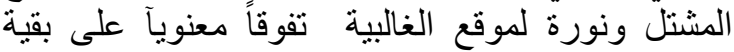

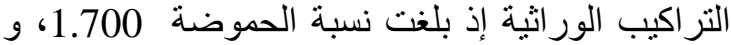

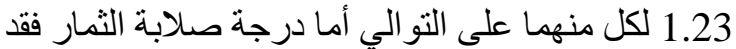

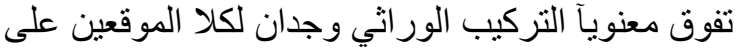

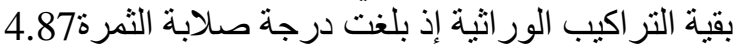

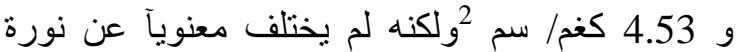
للموقعين و وعن وعد لموقع الغالبية ـ وتتفق هذه النتائج

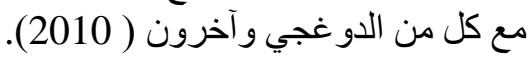

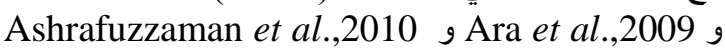

$$
E . G . A .=\frac{G \cdot A \cdot}{\bar{X}} X 100
$$

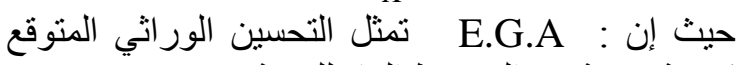
كنسبة مئوية من المتوسط العام للصفة. يمثل التحسين الوراثي المنوقع و الصفة وحسبت المديات التي اقترحسها (Agrwal1982)

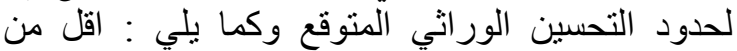

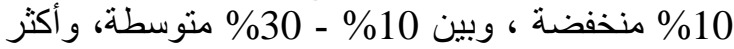
من 30\% عالية .وتم تقدير قيم معاملات الاختلاف المظهري و الور اثي وحسب المعادلات التالية :-

$$
\begin{aligned}
& P . C . V . \%=\frac{\sigma_{P}}{-} X 100 \\
& \text { G.C..V. }=\frac{\sigma_{G}}{-} X 100
\end{aligned}
$$

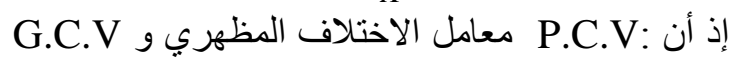

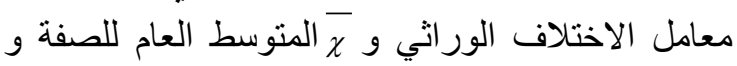

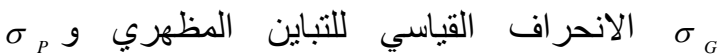
الانحر اف القياسي للتباين الور اثي التي الثبات المظهري (الاستقر ارية) Homeostasis $(\mathrm{H}) \%=1-(\mathrm{S} / \bar{X})$

$$
S=\sqrt{\frac{\sum X_{i}^{2}-\frac{\left(\sum x_{i}\right)^{2}}{n}}{n-1}}
$$

G.R = Genetic Resultant - : المحصلة الور اثية

$$
\mathrm{G} . \mathrm{R}=(\mathrm{H} \%) \frac{\text { Mean of cultivar }}{\text { Mean of cultivars }}
$$

\begin{tabular}{|c|c|c|c|c|c|c|c|c|}
\hline درجة صلابة & الحموضة & $\begin{array}{c}\text { الصبلة الذائبة } \\
\text { TSS } \\
\text { TSS }\end{array}$ & حاصل & الثرنة & عدد الثمار & المواقع & لدرجية & الاختلاف \\
\hline 0.715 & 0.229 & 0.061 & 0.115 & 24.20 & 101.27 & المشتل & 2 & \multirow{2}{*}{ مكررات } \\
\hline 0.126 & 0.350 & 2.117 & 0.334 & 40.27 & 46.07 & الغالبية & 2 & \\
\hline $1.345^{* * *}$ & $\mathbf{0 . 2 0 3}^{* *}$ & $4.442 *$ & $2.147 * *$ & 99.90* & 151.10** & المشتل & 4 & \multirow{2}{*}{ التراثية } \\
\hline $0.964 * *$ & $0.112^{* * *}$ & 1.792 & $2.102 * *$ & 172.27* & $174.60 * *$ & الغالبية & 4 & \\
\hline 0.168 & 0.021 & 0.725 & 0.175 & 23.45 & 14.60 & المشتل & 8 & \multirow{2}{*}{ التجريبي } \\
\hline 0.079 & 0.011 & 0.742 & 0.085 & 26.77 & 19.90 & الغالبية & 8 & \\
\hline
\end{tabular}

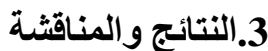

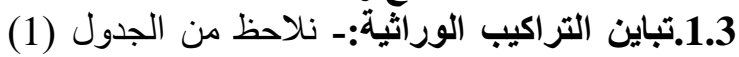

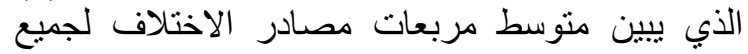

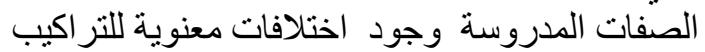

جدول (1): متوسطات المربعات (التباينات )للصفات المدروسة في الموقعين (المشتل ) ، (الغالبية). 


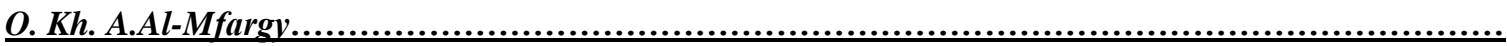

جذول (2): متوسطات الصفات المدروسة للتراكيب الوراثية للموقعين ( المشتل ، الغالبية ) .

\begin{tabular}{|c|c|c|c|c|c|c|c|}
\hline درجة صلابة & الحموضة & الصلبة الذائبة المواد & النبات & الثمرة & عدد الثمار & المواقع & التر اثيبة \\
\hline $\begin{array}{l}4.87 \\
4.53\end{array}$ & $\begin{array}{l}1.37 \\
0.73\end{array}$ & $\begin{array}{l}7.67 \\
6.00\end{array}$ & $\begin{array}{l}3.920 \\
3.754\end{array}$ & $\begin{array}{l}59.3 \\
60.3\end{array}$ & $\begin{array}{l}66.33 \\
62.30\end{array}$ & الغالبية & وجدان \\
\hline $\begin{array}{l}3.43 \\
3.27\end{array}$ & $\begin{array}{l}1.70 \\
1.07\end{array}$ & $\begin{array}{l}5.83 \\
5.33\end{array}$ & $\begin{array}{l}5.020 \\
5.191\end{array}$ & $\begin{array}{l}70.3 \\
73.0\end{array}$ & $\begin{array}{l}71.33 \\
71.00\end{array}$ & الغالبية & دينا \\
\hline \begin{tabular}{|l|}
3.93 \\
4.13
\end{tabular} & $\begin{array}{l}1.17 \\
1.00\end{array}$ & $\begin{array}{l}7.27 \\
6.17\end{array}$ & $\begin{array}{l}4.080 \\
3.948\end{array}$ & \begin{tabular}{|l|}
61.3 \\
56.3
\end{tabular} & $\begin{array}{l}66.67 \\
70.30\end{array}$ & الغالبية & وعد \\
\hline \begin{tabular}{|l|}
3.23 \\
3.30
\end{tabular} & \begin{tabular}{|l|}
1.03 \\
1.17
\end{tabular} & $\begin{array}{l}6.50 \\
4.33\end{array}$ & $\begin{array}{l}\mathbf{5 . 9 5 0} \\
\mathbf{5 . 3 4 2}\end{array}$ & $\begin{array}{l}71.0 \\
64.3\end{array}$ & $\begin{array}{l}83.67 \\
83.00\end{array}$ & الفالبية & شهيرة \\
\hline $\begin{array}{l}4.37 \\
4.17\end{array}$ & $\begin{array}{l}1.47 \\
1.23\end{array}$ & $\begin{array}{l}4.60 \\
4.83\end{array}$ & $\begin{array}{l}5.250 \\
5.540\end{array}$ & $\begin{array}{l}71.0 \\
73.3\end{array}$ & $\begin{array}{l}74.33 \\
76.00\end{array}$ & الغالبية & نورة \\
\hline \begin{tabular}{|l|}
3.97 \\
3.88 \\
\end{tabular} & $\begin{array}{l}1.35 \\
1.04\end{array}$ & $\begin{array}{l}6.37 \\
5.33\end{array}$ & $\begin{array}{l}4.840 \\
4.755 \\
\end{array}$ & $\begin{array}{l}66.6 \\
65.5\end{array}$ & $\begin{array}{l}72.47 \\
72.50 \\
\end{array}$ & الغالبية & المتوسط العام \\
\hline $\begin{array}{c}0.772 \\
0.5303\end{array}$ & $\begin{array}{l}0.2739 \\
0.1960\end{array}$ & $\begin{array}{l}1.603 \\
1.622\end{array}$ & $\begin{array}{l}0.789 \\
0.549\end{array}$ & $\begin{array}{l}9.12 \\
9.74\end{array}$ & $\begin{array}{l}7.194 \\
8.400\end{array}$ & الغشالبية & $\begin{array}{c}\text { L.S.D } \\
0.05\end{array}$ \\
\hline
\end{tabular}

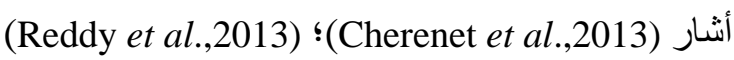
إلى انخفاض معامل الاختلاف الوراثي و المظهري

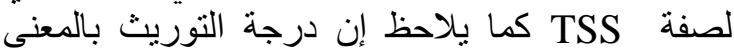

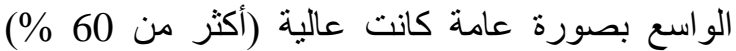
لأغلب الصفات ولكلا الموقعين وسيكون من السهولة التهونة انتخاب هذه الصفات لأنه سيكون هنالك تطابق وثيق التيق بين

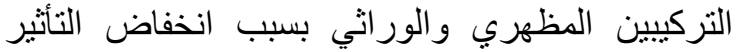

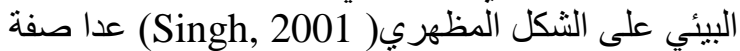

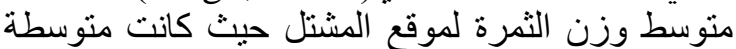

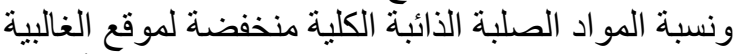
و التي تتفق معDeshmukh et al.,2005 كما أن قيم الغية النية نسبة التحسين الوراثي المتوقع حسب 1982 Agrwal

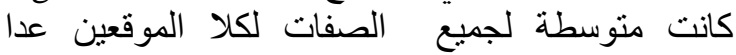
متوسط وزن الثمرة ونسبة المواد الصلبة الذائبة الكلية

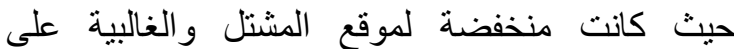
التوالي, تتفق هذه النتيجة مع كل من خليل (2008)

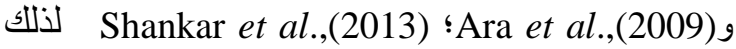
فإن الصفات التي تتميز بارتفاع قيم التوريث أدت إلى إلى

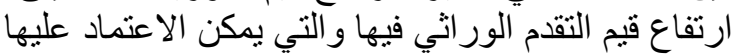

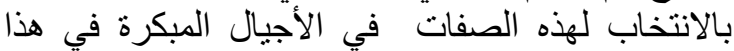

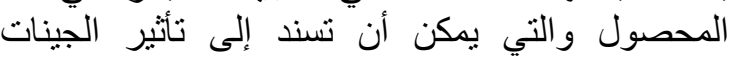
المضافة و عليه ممكن تحسينها بالانتخاب البـ Oskita and Adedolapo (2014) تشير النتائج الواردة في الجدول ( 4) إلى إنى وجود

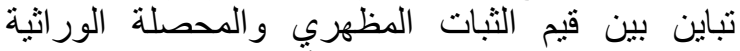
للصفات المدروسة إذ نلاحظ أن صفات أن وات عدد الثات الثمار ومتوسط وزن الثرة وحاصل النبات ذات ذات قيم عالية

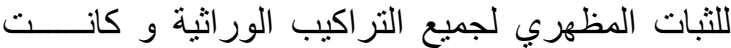

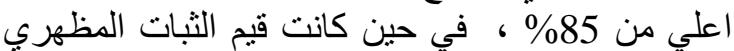

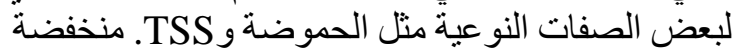

Shankar et al.,2013 و Reedy et al.,2013و

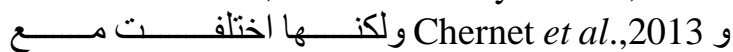
(2012) Osekita and Adedolapo(2014) حيث لم يلاحظ اختلافات معنوية بين الهجن في صفات

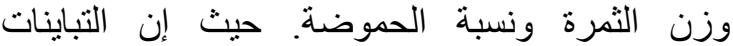

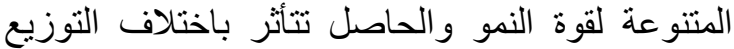
البيئي لأصناف الطماطم (Olaniyi et al.,2010 ). وقد يرجع التباين إلى الاختلافات الوراثية بين

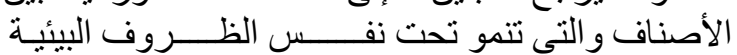

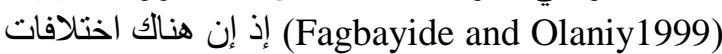

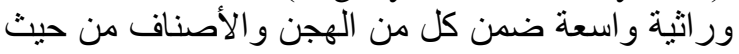

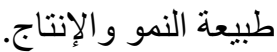
2.3.تقدير المعالم الوراثية تبين النتائج الواردة في جدول الودير (3) إلى أن التباين

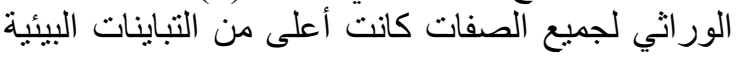

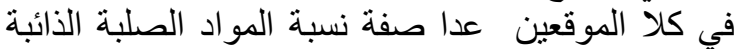

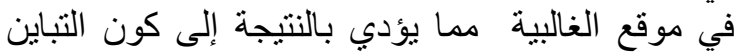

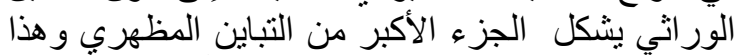

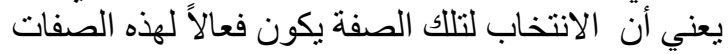

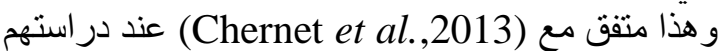
للتباينات الور اثية لصفات عدد الثمار و الحاصل للنبات

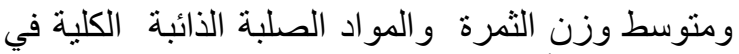
الطماطم من أن التباينات الور اثثية لهذه الصفات التهات نشكل

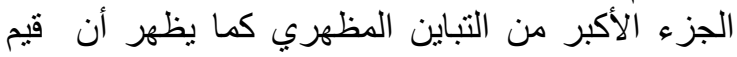

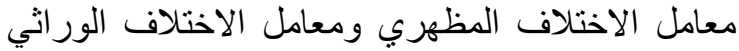

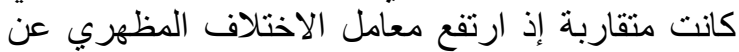

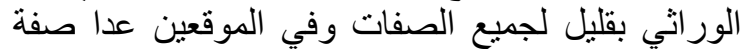

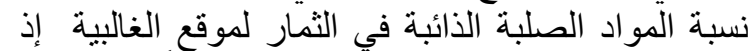

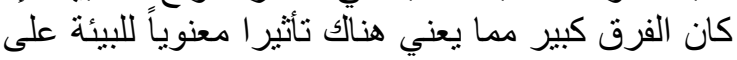

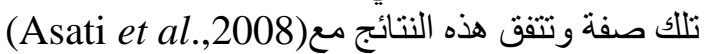
(Shanka et al.,2013) (Ara et al.,2009) 
جدول(3) : تقديرات المعالم الوراثية للصفات المدروسة ولكلا الموقعين .

\begin{tabular}{|c|c|c|c|c|c|c|c|}
\hline درجة صلابة & الحموضة & TSS\% & حاصل النبات & وزن الثمرة & عدد الثمار & المواقع & الوراثية \\
\hline 0.39 & 0.06 & 1.24 & 0.66 & 25.48 & 45.5 & المشتل & \multirow{2}{*}{$\sigma^{2} g$} \\
\hline 0.30 & 0.03 & 0.35 & 0.67 & 48.5 & 51.57 & الغالبية & \\
\hline 0.168 & 0.021 & 0.725 & 0.175 & 23.45 & 14.60 & المشتل & \multirow{2}{*}{$\sigma^{2} e$} \\
\hline 0.079 & 0.011 & 0.742 & 0.085 & 26.77 & 19.90 & الغالبية & \\
\hline 0.56 & 0.081 & 1.97 & 0.835 & 48.94 & 60.1 & المشتل & \multirow{2}{*}{$\sigma^{2} p$} \\
\hline 0.38 & 0.041 & 1.09 & 0.755 & 75.27 & 71.47 & الغالبية & \\
\hline 15.73 & 18.14 & 17.48 & 16.78 & 7.58 & 9.30 & المشتل & \multirow{2}{*}{ G.cv\% } \\
\hline 14.12 & 16.65 & 11.10 & 17.21 & 10.63 & 9.91 & الغالبية & \\
\hline 18.85 & 21.08 & 22.03 & 18.01 & 10.47 & 10.70 & المشتل & \multirow{2}{*}{ P.cv\% } \\
\hline 15.89 & 19.47 & 19.59 & 19.27 & 12.98 & 11.64 & الغالبية & \\
\hline 69.64 & 74.07 & 62.94 & 86.84 & 52.41 & 75.71 & المشتل & \multirow{2}{*}{ H b.s\% } \\
\hline 78.95 & 73.17 & 32.11 & 79.76 & 64.43 & 72.46 & الغالبية & \\
\hline 0.72 & 0.29 & 1.22 & 1.05 & 5.08 & 8.14 & المشتل & \multirow{2}{*}{ G.A } \\
\hline 0.67 & 0.21 & 0.46 & 1.01 & 7.77 & 8.50 & |الغالبية & \\
\hline 18.13 & 21.53 & 19.15 & 21.69 & 7.63 & 11.23 & المشتل & \multirow{2}{*}{ G.A\% } \\
\hline 17.27 & 20.19 & 8.63 & 21.24 & 11.86 & 11.72 & الغالبية & \\
\hline
\end{tabular}

جدول (4): قيم الثبات المظهري والمحصلة الوراثية للصفات المدروسة في الموقعين

\begin{tabular}{|c|c|c|c|c|c|c|c|}
\hline درجة صلابة & الحموضة & الصلبة الذائبة & حاصل & وزنمة & الثمار & لمدروسة & الصا \\
\hline 0.865 & 0.650 & 0.811 & 0.934 & 0.918 & 0.931 & H & \multirow[t]{2}{*}{ وجدان } \\
\hline 1.010 & 0.572 & 0.939 & 0.747 & 0.832 & 0.792 & GR & \\
\hline 0.893 & 0.666 & 0.818 & 0.928 & 0.951 & 0.963 & $\mathbf{H}$ & \multirow{2}{*}{ دينا } \\
\hline 0.770 & 0.772 & 0.785 & 0.985 & 1.032 & 0.948 & GR & \\
\hline 0.863 & 0.736 & 0.838 & 0.955 & 0.914 & 0.913 & H & \multirow[b]{2}{*}{ وعد } \\
\hline 0.897 & 0.668 & 0.947 & 0.798 & 0.814 & 0.852 & GR & \\
\hline 0.934 & 0.756 & 0.730 & 0.882 & 0.933 & 0.923 & H & \multirow[t]{2}{*}{ شهيرة } \\
\hline 0.789 & 0.697 & 0.670 & 1.038 & 0.956 & 1.064 & GR & \\
\hline 0.897 & 0.833 & 0.850 & 0.946 & 0.904 & 0.922 & H & \multirow{2}{*}{ نورة } \\
\hline 0.974 & 0.942 & 0.709 & 1.064 & 0.988 & 0.959 & GR & \\
\hline
\end{tabular}

أنبات المظهري ، GR

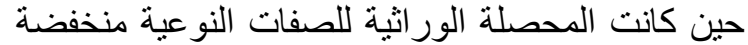

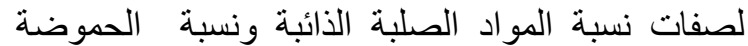
ودرجة صلابة الثمار للتركيبين الور اثيين دينا وشهيرة و هذه النتائج متو افقة مع زنتي وحسين (2011) .
باستثناء درجة الصلابة كانت مرتفعة. كما أن قيم المحصلة الور اثية كانت عالية للصفات الكمية في معظم كانة

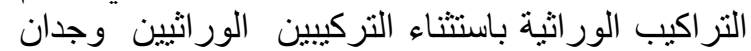

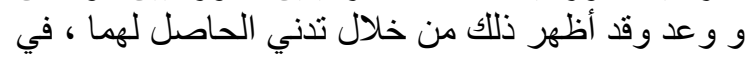




\section{REFERENCES}

Agrwal V. A. (1982). Heritability and genetic advance in Triticale . Indian Agric. Res.,16:19 $-23$.

Allard R. W. (1960). Principles of Plant Breeding. John willy and Sons, New York, USA. pp. 485.

Ara A., R. Narayan, N. Ahmed and Khan, S. H.(2009). Genetic variability and selection parameters for yield and quality attributes in tomato. Indian J. Hort. 66 (1): 73-78.

Asati B .S. , Rain N. and Singh A.K. (2008). Genetic parameters study for yield and quality traits in tomato . Asian J. Hort., 3 (2): 222-225.

Ashrafuzzaman M. A., Haque M. , Razi Ismail M.T., Islam and Shahidullah S.M. (2010) . Genotypic and seasonal variation in plant development and yield attributese in tomato (Lycopersicom esculentum Mill.) cultivars. International J. of Bot.6 (1):41-46.

Chernet S., Belew D. and Abay F. (2013). Genetic variability and association of characters in tomato (Solanum lycopersicon L.)genotypes in northern ethiopia. Int.'L J. Agric. Res., 8: 67-76.

Deshmukh S.N., Basu M.S. and Reddy P.S. (2005). Genetic variability, character association and path coefficient analysis of quantitative traits in viginia bunch varieties of groundnut. Indian J. Agric. Sci., 56: 515-518.

Gomez K.A. and Gomez A.A.(1984).Statistical Procedures for Agricultural Research, $2^{\text {nd }}$ ed.,John Wiley and Sons, New York, USA.

Hanson C .H., Robinson H.F., Comstock R.E. (1956). The biometrical studies on yield in segregating population of (korian lespedeza). Agron. J. 48: 268-272.

Kempthorne B. (1969) . An Introduction to genetic statistics . Ames . Iowa State. Univ. Press, USA.

Olaniyi J.O., Akanbi W.B., Adejumo T.A. and Akande O.G (2010). Growth, fruit yield and nutritional quality of tomato varieties.

Afr. J. Food Sci . 4 (6) : 398-402 .

Olaniyi J.O. and Fagbayide J.A. (1999) . Performance of eight $F_{1}$ Hybrid cabbage (Brassica olerácea L.) varieties in the Southern Guinea Savanna zone of Nigeria. J. Agric. Biotech. Environ., 1: 4-10.

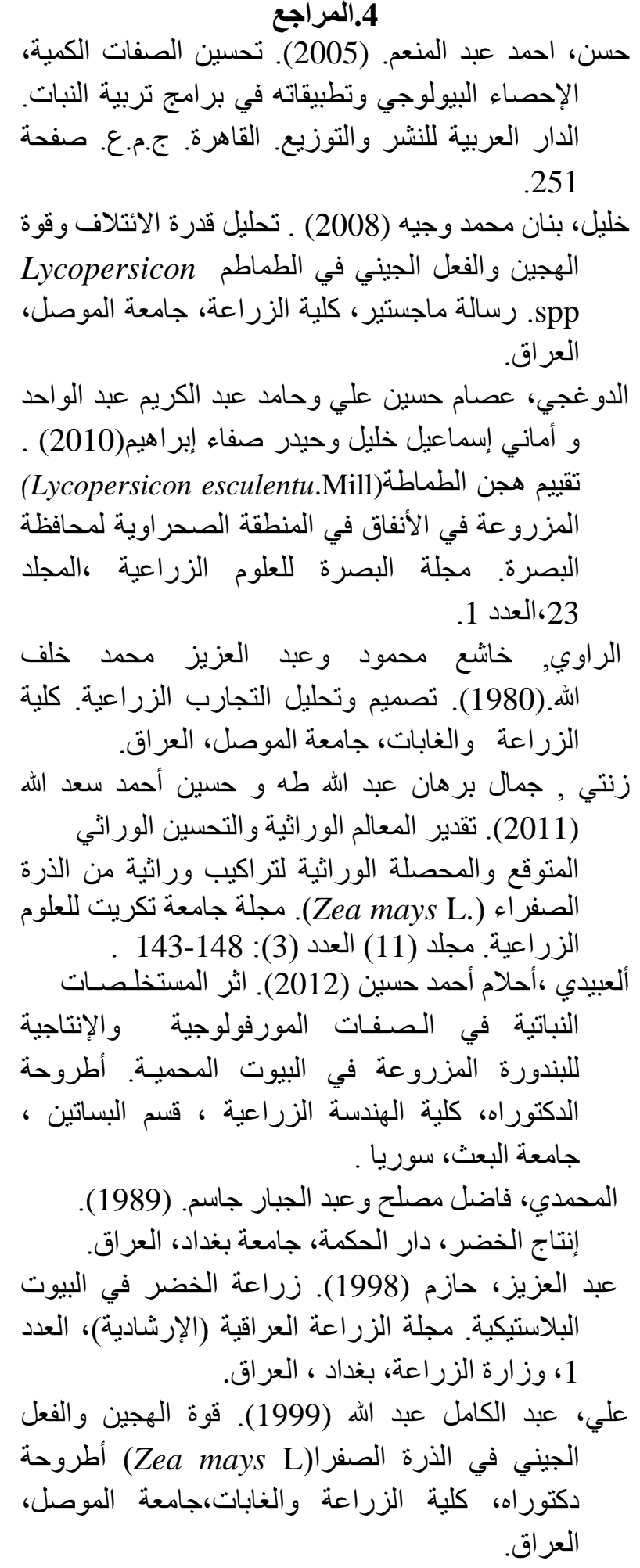

حسن، احمد عبد المنعم. (2005). تحسين الصفات الكمية، الإحصاء البيولوجي وتطبيقاته في برامج نربية النبات 251

خليل، بنان محمد وجيه (2008) ـ تحليل قدرة الائتلاف وقوة

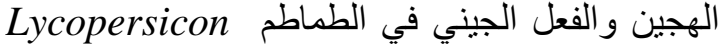
נ.spp

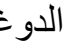
و أماني إسماعيل خليل وحيدر صفاء إبر اهيم(2010) . تفييم هجن الطماطة(Lycopersicon esculentu.Mill المزرو عة في الأنفاق في المنطقة الصحر اوية لمحافظة

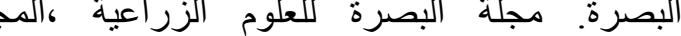

الراوي, خاشع محمود وعبد العزيز محمد خلف

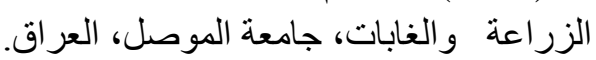

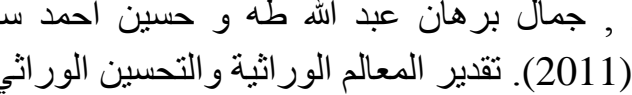

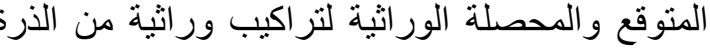
الصفر اء (Zea mays L) (11) مجلة جامعة تكريت للعلوم

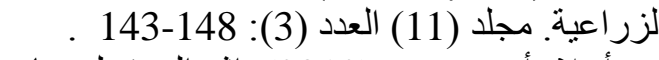
النباتية في الصفـات المورفولوجية و والإنتاجية

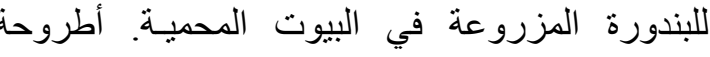
الدكتور اه، كلية الهندسة الزراعية ، قسم البساتين ، المحمدي، فاضل مصلح و عبد الجبار جاسم. (1989).

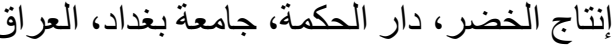

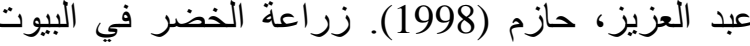

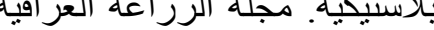


Oskita O.S. and Adedolapo T.A. (2014) Genetic of advance heritability and character association of component of yield in some genotypes of tomato (Lycopersicon Esculentum Mill.).Wettsd cademia, J. Biotech. 2(1): 6-10.

Ranganna S. (1977).Handbook of analysis and quality control for fruit and vegetable products $2^{\text {nd }}$ copyright by Tata McGraw- Hill publishing company limited .P.1103.

Reddy B. R., Reddy, K.Reddaiah D. S. and Sunil N. (2013). Studies on genetic variability, heritability and genetic advance for yield and quality traits in tomato (Solanum lycopersicum L.) Int. J. Curr . Microbiol .App . Sci. 2(9): 238-244

Scapim C.A., Oliveira V.R., Braccini A.L., Cruz C.D., Andrade C.A.B. and Vidigal M.C.G.
(2000). Yield stability in Maize (Zea mays L.) and correlations among the parameters of the eberhart and russel, lin and binns and huehn models. Genet. Mol. Biol. 23.2.

Shankar A., Reddy R.V.S.K. , Sujatha M. and Pratap M. (2013). Genetic variability studies in $\mathrm{F}_{1}$ generation of tomato (Solanum lycopersicon L.) IOSR Journal of Agric. and Vet. Sci . 4 (5): 31-34.

Singh B.D. (2001). Plant breeding principles and methods. Kalyani Publishers, New Delhi, India,. P: 896.

Worku M., Zelleke H., Taye G., Tolessa B., Wolde L., Abera W., Guta A. and H. Tuna (2001). Yield stability of Maize (Zea mays L.) genotypes across locations. seventh eastern and south. Africa Regional Maize Conference, p. 139-142. 